16

\title{
Об электронной эмиссии из фокальной области симметрично сходящейся поверхностной плазмонной волны на свободной поверхности металлической пленки
}

\author{
(C) А.Б. Петрин \\ Объединенный институт высоких температур РАН, \\ 125412 Москва, Россия \\ e-mail: a_petrin@mail.ru
}

Поступила в редакцию 27.02.2020 г.

В окончательной редакции 27.02.2020 г.

Принята к публикации 15.03.2020 г.

На основе теории отражения плоской электромагнитной волны от плоскослоистой структуры рассмотрены возбуждение и фокусировка радиально сходящейся поверхностной плазмонной волны на свободной поверхности золотой пленки в схеме Кречмана. Количественно исследовано распределение электрического поля в окрестности фокуса на свободной поверхности пленки. На основе этого распределения и теории термополевой электронной эмиссии из металла проведена оценка среднего по времени тока электронной эмиссии в зависимости от амплитуды нормальной составляющей электрического поля волны в центре фокального распределения. Показано, что основная часть эмиссионного тока исходит из области вокруг центра фокуса диаметром порядка десятой части рабочей длины волны в вакууме, причем из окружающих центральный максимум дифракционных колец исходит пренебрежимо малая часть эмиссионного тока.

Ключевые слова: нанофокусировка, поверхностные плазмоны, оптические сенсоры.

DOI: $10.21883 /$ OS.2020.08.49719.68-20

\section{Введение и постановка задачи}

В последнее время значительные усилия были направлены на развитие теоретических методов исследования возбуждения поверхностных волн ограниченными световыми пучками в схеме Кречмана. В частности, была обнаружена нелокальность отражения, сопровождающаяся возбуждением поверхностных волн [1]. Исследования позволили теоретически объяснить эффект Гуса-Хенхена, проявляющийся в расщеплении отраженной волны [2], а также рассмотреть интерференцию поверхностных волн [3]. Дальнейшие развитие этого направления позволило разработать схему оптимальной фокусировки поверхностной, сходящейся в точку на поверхности, и вычислить распределение электрического поля в поверхностном фокусе [4].

В настоящей работе полученные результаты применены к теоретическому рассмотрению электронной эмиссии, стимулированной оптической волной в области фокуса. Само явление электронной эмиссии с поверхности металлической пленки, стимулированное поверхностной плазмонной волной, наблюдалось экспериментально $[5,6]$. Однако возможность фокусировки поверхностной плазмонной волны на свободной поверхности металлической пленки позволило бы получать оптически стимулированную электронную эмиссию из локальных областей на поверхности. Более того, можно предположить, что эта область поверхностной электронной эмиссии в силу крайне сильной зависимости плотности тока эмиссии от напряженности поля на поверхности может быть заметно меньше длины световой волны. Последнее обстоятельство дало бы возможность, например, исследовать эмитирующую способность поверхности пленки с повышенным разрешением или создавать специальные локализованные эмитирующие электроны источники для электронно-оптических систем. Исследованию этого круга задач и посвящена настоящая работа.

Конкретно в данной работе теоретически рассматривается задача оптимального возбуждения радиально сходящейся поверхностной плазмонной волны на свободной границе пленки металла. Идея решения этой задачи основана на исследовании возбуждения поверхностной плазмонной волны в схеме Кречмана (рис. 1) оптическими пучками ограниченной апертуры [3]. Оказалось (рис. 4, $b$ работы [3]), что ширина падающего пучка в схеме Кречмана должна быть достаточно велика. Это необходимо, чтобы поверхностная волна выросла до максимально возможной амплитуды.

В рамках обычной схемы Кречмана [7] (рис. 1) согласование поверхностной плазмонной волны 1 на поверхности металлической пленки 2 и падающей волны 3 осуществляется с помощью стеклянной призмы 4. Линейно поляризованная плоская монохроматическая волна $\mathbf{E}^{i}$ падает на металлическую пленку со стороны призмы. Вектор напряженности электрического поля падающей волны направлен в плоскости падения ( $p$-поляризация). Призма необходима для согласования падающей плоской волны и поверхностной плазмонной волны по вол- 


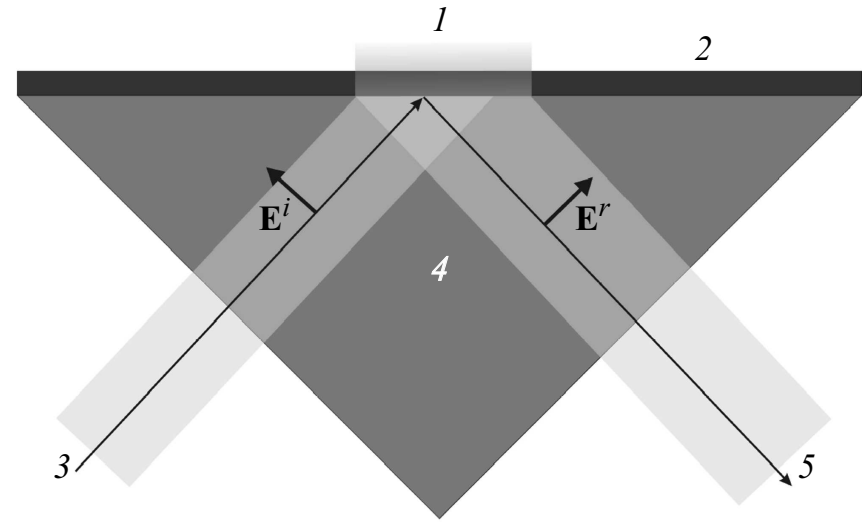

Рис. 1. Возбуждение поверхностных плазмонов 1 на поверхности металлической пленки 2 по схеме Кречмана. Падающая волна 3 в призме 4 порождает поверхностную плазмонную волну 1 и отраженную волну 5 .

новому числу. Волновое число поверхностной плазмонной волны больше волнового числа плоской волны в свободном пространстве той же частоты $[8,9]$. Чтобы обеспечить равенство волновых чисел падающей 3 , отраженной 5 и поверхностной плазмонной 1 волн вдоль поверхности металла (рис. 1), необходимо, чтобы падающая волна подходила к пленке из оптически более плотной среды (где волновое число больше, чем в свободном пространстве). Показатель преломления призмы и угол падения подбираются таким образом, чтобы обеспечивалось равенство волновых чисел падающей и поверхностных волн. При этих параметрах в отсутствие металлической пленки (и возбуждения поверхностных плазмонов) наблюдается полное внутреннее отражение падающей волны.

Экспериментальные и теоретические исследования показывают, что, если угол падения соответствует согласованию по волновому числу падающей и поверхностной плазмонной волн, наблюдается резкое падение коэффициента отражения. Например, при длине падающей волны в вакууме $\lambda=633 \mathrm{~nm}$, показателя преломления призмы $n_{p}=1.6$ и толщине пленки золота приблизительно равной $48.7 \mathrm{~nm}$ (эта величина зависит от конкретного значения показателя преломления призмы и частоты падающей волны) наблюдается сильное изменение коэффициента отражения практически от единицы до нуля в окрестности угла падения, соответствующего согласованию свободной и поверхностной плазмонной волн $\alpha=41.0^{\circ}$. При небольшом отклонении угла падения от угла согласования $\alpha$ коэффициент отражения возвращается практически от нуля к единичному значению. Диэлектрическая проницаемость золота на длине волны $\lambda=633 \mathrm{~nm}$ принималась равной $\varepsilon_{m}=-11.6+i 1.2[10]$.

Поле поверхностной плазмонной волны сосредоточено в тонком слое вблизи поверхности металла, поэтому характер отражения в схеме Кречмана сильно зависит от изменений показателя преломления в тонком слое вблизи поверхности. Именно на этом основывается широкое применение схемы Кречмана для создания различного рода датчиков, обладающих высокой чувствительностью [11].

Возникновение минимума отражения при возбуждении поверхностной волны сопровождается максимумом амплитуды поверхностной волны на внешней границе пленочной структуры с возбужденной поверхностной волной. Эта амплитуда намного больше амплитуды падающей волны.

В настоящей работе задача точечной фокусировки на свободной поверхности пленки решается посредством создания радиально сходящейся поверхностной волны, возбужденной специальной конической волной. Поверхностная плазмонная стоячая волна 1 (рис. 2) возбуждается на поверхности золотой пленки 2 с помощью конической падающей волны 3 в конической призме 4. Падающая 3 и отраженная 5 конические волны имеют $p$-поляризацию, т. е. вектор напряженности электрического поля лежит в плоскости, проходящей через ось $Z$. Мы будем интересоваться распределением поля сфокусированной волны на свободной поверхности металлической пленки 2 и значением амплитуды поля в фокусе, а затем вычислим ток электронной эмиссии со свободной поверхности пленки.

\section{Общая задача падения электромагнитной волны на плоскослоистую структуру}

Рассмотрим задачу падения монохроматической волны с циклической частотой $\omega$ единичной амплитуды из однородного полупространства (призмы), заполненного однородным изотропным диэлектриком с показателем преломления $n_{1}$, на плоскую пленку толщины $d_{1}$. Предполагается для определенности, что пленка металличе-

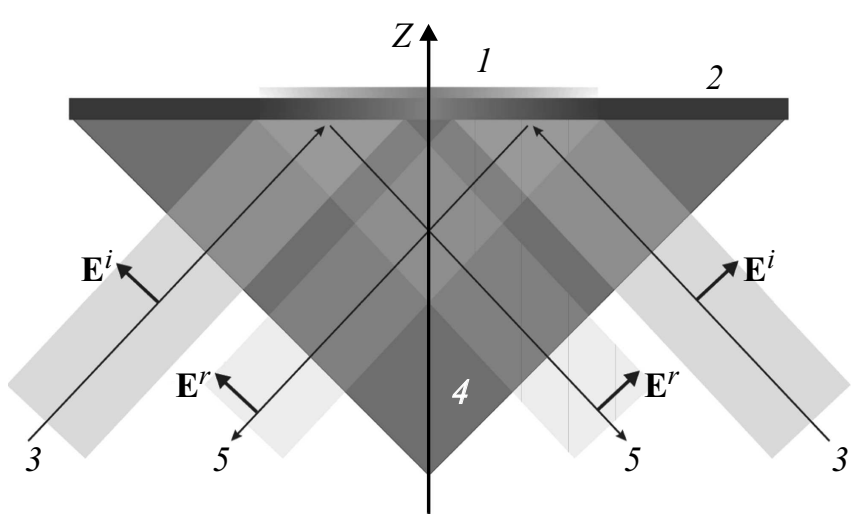

Рис. 2. Возбуждение поверхностной плазмонной стоячей волны 1 на поверхности серебряной пленки 2 с помощью конической волны 3 , падающей со стороны конической призмы 4. Падающая 3 и отраженная 5 конические волны имеют p-поляризацию. 
ская с комплексной диэлектрической проницаемостью $\varepsilon_{2}$ на рассматриваемой частоте $\omega$, а за пленкой находится однородное полупространство с диэлектрической проницаемостью $\varepsilon_{3}$ (свободное пространство). Сначала будет рассмотрена задача с одной пленкой, а затем она будет обобщена на многопленочную структуру с произвольным количеством пленок. Данный подход к решению электромагнитной задачи для многопленочной структуры восходит к работе А. Зоммерфельда [12] и развит в работах $[10,13]$.

Уравнения Максвелла в рассматриваемых областях можно записать в виде

$$
\begin{gathered}
\operatorname{rot} \mathbf{E}_{j}=i \omega \mathbf{B}_{j}, \\
\operatorname{rot} \mathbf{B}_{j}=-i \omega \varepsilon_{j} \mu_{j} \mathbf{E}_{j},
\end{gathered}
$$

где $\mathbf{E}_{j}$ и $\mathbf{B}_{j}$ - векторы напряженности электрического поля и индукции магнитного поля в области с номером $j ; j=1$ соответствует полупространству 1 , для которого $z<0 ; j=2$ соответствует области пленки 2 $\left(0<z<d_{1}\right) ; j=3$ соответствует свободному полупространству $(z>d)$. Комплексное представление во времени предполагается в виде $e^{-i \omega t}$.

Из уравнений (1) и (2) следует, что электрическое и магнитное поля удовлетворяют однородному волновому уравнению, т.е.

$$
\begin{aligned}
& \operatorname{rot} \operatorname{rot} \mathbf{E}_{j}-\omega^{2} \mu_{j} \varepsilon_{j} \mathbf{E}_{j}=0, \\
& \operatorname{rot} \operatorname{rot} \mathbf{B}_{j}-\omega^{2} \mu_{j} \varepsilon_{j} \mathbf{E}_{j}=0 .
\end{aligned}
$$

Учитывая, что $\operatorname{div} \mathbf{D}_{j}=0 \quad$ или $\partial_{x} E_{j, x}+\partial_{y} E_{j, y}+$ $+\partial_{z} E_{j, z}=0$, из (3) получаем уравнение для $E_{j, x}$ :

$$
\partial_{x x}^{2} E_{j, x}+\partial_{y y}^{2} E_{j, x}+\partial_{z z} E_{j, x}+\omega^{2} \mu_{j} \varepsilon_{j} E_{j, x}=0 .
$$

Аналогичные уравнения получаются для компонент напряженности электрического поля $E_{j, y}, E_{j, z}$ и индукции магнитного поля $B_{j, x}, B_{j, y}$ и $B_{j, z}$ (для краткости уравнения не выписываем).

Представим в (4) компоненты напряженности электрических полей в виде фурье-разложений:

$$
E_{j, x}(x, y, z)=\frac{1}{(2 \pi)^{2}} \int_{-\infty}^{\infty} \int_{-\infty}^{\infty} \tilde{E}_{j, x}(\xi, \eta, z) e^{i(\xi x+\eta y)} d \xi d \eta
$$

плюс аналогичные выражения для $y$ - и $z$-компонент, где фурье-образы определяются интегралами:

$$
\tilde{E}_{j, x}(\xi, \eta, z)=\int_{-\infty}^{+\infty} \int_{-\infty}^{+\infty} E_{j, x}(x, y, z) e^{-i(\xi x+\eta y)} d x d y,
$$

и аналогично для $y$ - и $z$-компонент напряженности поля.

Тогда для фурье-образов получим уравнение для $\tilde{E}_{j, x}$ :

$$
\frac{d^{3} \tilde{E}_{j, x}}{d z^{2}}+\left(\omega^{2} \mu_{j} \varepsilon_{j}-\xi^{2}-\eta^{2}\right) \tilde{E}_{j, x}=0,
$$

и аналогичные уравнения для $\tilde{E}_{j, y}$ и $\tilde{E}_{j, z}$ (для краткости не выписываем).

Решением этих уравнений являются $\tilde{E}_{j, x}^{ \pm}=\hat{E}_{j, x}^{ \pm} e_{j, z}^{ \pm i k} z$, $\tilde{E}_{j, y}^{ \pm}=\hat{E}_{j, y}^{ \pm} e^{ \pm i k_{j, z} z}, \tilde{E}_{j, z}^{ \pm}=\hat{E}_{j, k}^{ \pm} e^{ \pm i k_{j, z} z}$, где $\hat{E}_{j, x}, \hat{E}_{j, z}^{-}, \hat{E}_{j, y}^{+}$, $\hat{E}_{j, y}^{-}, \quad \hat{E}_{j, z}^{+}, \quad \hat{E}_{j, z}-$ функции только от $\quad \xi \quad$ и $\eta$; $k_{j, z}=k_{j, z}(\xi, \eta)=\sqrt{\omega^{2} \mu_{j} \varepsilon_{j}-\xi^{2}-\eta^{2}}$.

Тогда общее решение уравнений (5) для направлений распространения волн вдоль „,+“ и против „-““ оси $Z$ можно записать в виде

$$
\begin{aligned}
\mathbf{E}_{j}^{ \pm}(x, y, z)= & \frac{1}{(2 \pi)^{2}} \int_{-\infty}^{\infty} \int_{-\infty}^{\infty}\left(\begin{array}{c}
\hat{E}_{j, x}^{ \pm}(\xi, \eta) \\
\hat{E}_{j, y}^{ \pm}(\xi, \eta) \\
\hat{E}_{j, z}^{ \pm}(\xi, \eta)
\end{array}\right) e^{ \pm i k_{j, z} z} \\
& \times e^{i(\xi x+\eta y)} d \xi d \eta .
\end{aligned}
$$

Так как $\operatorname{div} \mathbf{D}_{j}=0, \quad$ то $\quad \varepsilon_{j} \xi \hat{E}_{j, x}+\varepsilon_{j} \eta \hat{E}_{j, y} \pm$ $\pm \varepsilon_{j} k_{j, x}(\xi) \hat{E}_{j, x}=0 \quad$ и общее решение (6) можно переписать в виде

$$
\begin{aligned}
\mathbf{E}_{j}^{ \pm}(x, y, z)= & \frac{1}{(2 \pi)^{2}} \int_{-\infty}^{\infty} \int_{-\infty}^{\infty}\left(\begin{array}{cc}
1 & 0 \\
0 & 1 \\
\mp \xi / k_{j, x} & \mp \eta / k_{j, x}
\end{array}\right) \\
& \times\left(\begin{array}{c}
\hat{E}_{j, x}^{ \pm}(\xi, \eta) \\
\hat{E}_{j, y}^{ \pm}(\xi, \eta)
\end{array}\right) e^{ \pm i k_{j, z} z} e^{i(\xi x+\eta y)} d \xi d \eta .
\end{aligned}
$$

Запишем общее решение в рассматриваемых областях (рис. 2) в следующем виде: в области 1

$$
\begin{aligned}
\mathbf{E}_{1}(x, y, z)= & \frac{1}{(2 \pi)^{2}} \int_{-\infty}^{+\infty} \int_{-\infty}^{+\infty}\left(\begin{array}{cc}
1 & 0 \\
0 & 1 \\
-\xi / k_{1, z} & -\eta / k_{1, z}
\end{array}\right) \\
& \times\left(\begin{array}{c}
\hat{E}_{1, x}^{+} \\
\hat{E}_{1, y}^{+}
\end{array}\right) e^{i k_{1, z} z} e^{i(\xi x+\eta y)} d \xi d \eta \\
& +\frac{1}{(2 \pi)^{2}} \int_{-\infty}^{+\infty} \int_{-\infty}^{+\infty}\left(\begin{array}{cc}
1 & 0 \\
0 & 1 \\
\xi / k_{1, z} & \eta / k_{1, z}
\end{array}\right) \\
& \times\left(\begin{array}{l}
\hat{E}_{1, x}^{-} \\
\hat{E}_{1, y}^{-}
\end{array}\right) e^{-i k_{1, z} z} e^{i(\xi x+\eta y)} d \xi d \eta,
\end{aligned}
$$


в области 2

$$
\begin{aligned}
\mathbf{E}_{2}(x, y, z)= & \frac{1}{(2 \pi)^{2}} \int_{-\infty}^{+\infty} \int_{-\infty}^{+\infty}\left(\begin{array}{cc}
1 & 0 \\
0 & 1 \\
-\xi / k_{2, z} & -\eta / k_{2, z}
\end{array}\right) \\
& \times\left(\begin{array}{c}
\hat{E}_{2, x}^{+} \\
\hat{E}_{2, y}^{+}
\end{array}\right) e^{i k_{2, z} z} e^{i(\xi x+\eta y)} d \xi d \eta \\
& +\frac{1}{(2 \pi)^{2}} \int_{-\infty}^{+\infty} \int_{-\infty}^{+\infty}\left(\begin{array}{cc}
1 & 0 \\
0 & 1 \\
\xi / k_{2, z} & \eta / k_{2, z}
\end{array}\right) \\
& \times\left(\begin{array}{l}
\hat{E}_{2, x}^{-} \\
\hat{E}_{2, y}^{-}
\end{array}\right) e^{-i k_{2, z}\left(z-d_{1}\right)} e^{i(\xi x+\eta y)} d \xi d \eta,
\end{aligned}
$$

в области 3

$$
\begin{aligned}
\mathbf{E}_{3}(x, y, z)= & \frac{1}{(2 \pi)^{2}} \int_{-\infty}^{+\infty} \int_{-\infty}^{+\infty}\left(\begin{array}{cc}
1 & 0 \\
0 & 1 \\
-\xi / k_{3, z} & -\eta / k_{3, z}
\end{array}\right) \\
& \times\left(\begin{array}{c}
\hat{E}_{3, x}^{+} \\
\hat{E}_{3, y}^{+}
\end{array}\right) e^{i k_{3, z}\left(z-d_{1}\right)} e^{i(\xi x+\eta y)} d \xi d \eta .
\end{aligned}
$$

Аналогичные выражения в трех областях можно написать и для магнитного поля.

Из уравнения $\operatorname{rot} \mathbf{E}_{j}=i \omega \mathbf{B}_{j}$ найдем $x$ - и $y$-компоненты индукции магнитного поля. Для соответствующих фурье-компонент найдем

$$
\begin{aligned}
\hat{\mathbf{B}}_{j}^{+}= & \mathbf{e}_{x}\left(\frac{\eta}{\omega} \hat{E}_{j, z}^{+}-\frac{k_{j, z}}{\omega} \hat{E}_{j, y}^{+}\right)-\mathbf{e}_{y}\left(\frac{\xi}{\omega} \hat{E}_{j, z}^{+}-\frac{k_{j, z}}{\omega} \hat{E}_{j, x}^{+}\right) \\
& +\mathbf{e}_{z}\left(\frac{\xi}{\omega} \hat{E}_{j, y}^{+}-\frac{\eta}{\omega} \hat{E}_{j, x}^{+}\right),
\end{aligned}
$$

где $\mathbf{e}_{x}, \mathbf{e}_{y}$ и $\mathbf{e}_{z}-$ единичные орты осей координат. Далее, учитывая, что ,

$$
\hat{E}_{j, z}^{+}=-\frac{\xi}{k_{j, z}} \hat{E}_{j, x}^{+}-\frac{\eta}{k_{j, z}} \hat{E}_{j, y}^{+},
$$

получим в матричном виде

$$
\left(\begin{array}{c}
\hat{B}_{j, x}^{+} \\
\hat{B}_{j, y}^{+} \\
\hat{B}_{j, z}
\end{array}\right)=\left[\begin{array}{cc}
-\xi \eta / \omega k_{j, z} & -\left(k_{j, z}^{2}+\eta^{2}\right) / \omega k_{j, z} \\
\left(k_{j, z}^{2}+\xi^{2}\right) / \omega k_{j, z} & \xi \eta / \omega k_{j, z} \\
-\eta / \omega & \xi / \omega
\end{array}\right]\left(\begin{array}{c}
\hat{E}_{j, x}^{+} \\
\hat{E}_{j, y}^{+}
\end{array}\right) .
$$

Аналогично для $\hat{\mathbf{B}}_{j}^{-}$найдем

$$
\left(\begin{array}{c}
\hat{B}_{j, x}^{-} \\
\hat{B}_{j, y}^{-} \\
\hat{B}_{j, z}
\end{array}\right)=\left[\begin{array}{cc}
\xi \eta / \omega k_{j, z} & \left(k_{j, z}^{2}+\eta^{2}\right) / \omega k_{j, z} \\
-\left(k_{j, z}^{2}+\xi^{2} / \omega k_{j, z}\right. & -\xi \eta / \omega k_{j, z} \\
-\eta / \omega & \xi / \omega
\end{array}\right]\left(\begin{array}{c}
\hat{E}_{j, x}^{-} \\
\hat{E}_{j, y}^{-}
\end{array}\right) .
$$

Тогда для тангенциальных компонент полей можно записать матричные соотношения:

$$
\left(\begin{array}{c}
\hat{B}_{j, x}^{+} \\
\hat{B}_{j, y}^{+}
\end{array}\right)=\left[\begin{array}{cc}
-\xi \eta / \omega k_{j, z} & -\left(k_{j, z}^{2}+\eta^{2}\right) / \omega k_{j, z} \\
\left(k_{j, z}^{2}+\xi^{2}\right) / \omega k_{j, z} & \xi \eta / \omega k_{j, z}
\end{array}\right]\left(\begin{array}{c}
\hat{E}_{j, x}^{+} \\
\hat{E}_{j, y}^{+}
\end{array}\right)
$$

и

$$
\left(\begin{array}{c}
\hat{B}_{j, x}^{-} \\
\hat{B}_{j, y}^{-}
\end{array}\right)\left[\begin{array}{cc}
\xi \eta / \omega k_{j, z} & \left(k_{j, z}^{2}+\eta^{2}\right) / \omega k_{j, z} \\
-\left(k_{j, z}^{2}+\xi^{2}\right) / \omega k_{j, z} & -\xi \eta / \omega k_{j, z}
\end{array}\right]\left(\begin{array}{c}
\hat{E}_{j, x}^{-} \\
\hat{E}_{j, y}^{-}
\end{array}\right) .
$$

Прежде чем записать граничные условия, выпишем тангенциальные электрические и магнитные поля: в области 1

$$
\begin{aligned}
& \mathbf{E}_{1, x}=\frac{1}{(2 \pi)^{2}} \int_{-\infty}^{+\infty} \int_{-\infty}^{+\infty}\left(\begin{array}{c}
\hat{E}_{1, x}^{+} \\
\hat{E}_{1, y}^{+}
\end{array}\right) e^{i k_{1, z} z} e^{i(\xi x+\eta y)} d \xi d \eta \\
& +\frac{1}{(2 \pi)^{2}} \int_{-\infty}^{+\infty} \int_{-\infty}^{+\infty}\left(\begin{array}{c}
\hat{E}_{1, x}^{-} \\
\hat{E}_{1, y}^{-}
\end{array}\right) e^{-i k_{1, z} z} e^{i(\xi x+\eta y)} d \xi d \eta,
\end{aligned}
$$

$\mathbf{B}_{1, \tau}=\frac{1}{(2 \pi)^{2}} \int_{-\infty}^{+\infty} \int_{-\infty}^{+\infty}\left(\begin{array}{cc}-\xi \eta / \omega k_{1, z} & -\left(k_{1, z}^{2}+\eta^{2}\right) / \omega k_{1, z} \\ \left(k_{1, z}^{2}+\xi^{2}\right) / \omega k_{1, z} & \xi \eta / \omega k_{1, z}\end{array}\right)$

$$
\begin{aligned}
& \times\left(\begin{array}{c}
\hat{E}_{1, x}^{+} \\
\hat{E}_{1, y}^{+}
\end{array}\right) e^{i k_{1, z} z} e^{i(\xi x+\eta y)} d \xi d \eta \\
& +\frac{1}{(2 \pi)^{2}} \int_{-\infty}^{+\infty} \int_{-\infty}^{+\infty}\left(\begin{array}{cc}
\xi \eta / \omega k_{1, z} & \left(k_{1, z}^{2}+\eta^{2}\right) / \omega k_{1, z} \\
-\left(k_{1, z}^{2}+\xi^{2}\right) / \omega k_{1, z} & -\xi \eta / \omega k_{1, z}
\end{array}\right)
\end{aligned}
$$$$
\times\left(\begin{array}{c}
\hat{E}_{1, x}^{-} \\
\hat{E}_{1, y}^{-}
\end{array}\right) e^{-i k_{1, z} z} e^{i(\xi x+\eta y)} d \xi d \eta
$$

в области 2

$$
\begin{aligned}
\mathbf{E}_{2, \tau}= & \frac{1}{(2 \pi)^{2}} \int_{-\infty}^{+\infty} \int_{-\infty}^{+\infty}\left(\begin{array}{c}
\hat{E}_{2, x}^{+} \\
\hat{E}_{2, y}^{+}
\end{array}\right) e^{i k_{2, z} z} e^{i(\xi x+\eta y)} d \xi d \eta \\
& +\frac{1}{(2 \pi)^{2}} \int_{-\infty}^{+\infty} \int_{-\infty}^{+\infty}\left(\begin{array}{c}
\hat{E}_{2, x}^{-} \\
\hat{E}_{2, y}^{-}
\end{array}\right) e^{-i k_{2, z}\left(z-d_{1}\right)} e^{i(\xi x+\eta y)} d \xi d \eta,
\end{aligned}
$$




$$
\begin{aligned}
& \mathbf{B}_{2, \tau}=\frac{1}{(2 \pi)^{2}} \int_{-\infty}^{+\infty} \int_{-\infty}^{+\infty}\left(\begin{array}{cc}
-\xi \eta / \omega k_{2, z} & -\left(k_{2, z}^{2}+\eta^{2}\right) / \omega k_{2, z} \\
\left(k_{2, z}^{2}+\xi^{2}\right) / \omega k_{2, z} & \xi \eta / \omega k_{2, z}
\end{array}\right) \\
& \times\left(\begin{array}{c}
\hat{E}_{2, x}^{+} \\
\hat{E}_{2, y}^{+}
\end{array}\right) e^{i k_{2, z} z} e^{i(\xi x+\eta y)} d \xi d \eta \\
& +\frac{1}{(2 \pi)^{2}} \int_{-\infty}^{+\infty} \int_{-\infty}^{+\infty}\left(\begin{array}{cc}
\xi \eta / \omega k_{2, z} & \left(k_{2, z}^{2}+\eta^{2}\right) / \omega k_{2, z} \\
-\left(k_{2, z}^{2}+\xi^{3}\right) / \omega k_{2, z} & -\xi \eta / \omega k_{2, z}
\end{array}\right) \\
& \times\left(\begin{array}{l}
\hat{E}_{2, x}^{-} \\
\hat{E}_{2, y}^{-}
\end{array}\right) e^{-i k_{2, z}\left(z-d_{1}\right)} e^{i(\xi x+\eta y)} d \xi d \eta,
\end{aligned}
$$

в области 3

$$
\begin{aligned}
& \mathbf{E}_{3, \tau}=\frac{1}{(2 \pi)^{2}} \int_{-\infty}^{+\infty} \int_{-\infty}^{+\infty}\left(\begin{array}{c}
\hat{E}_{3, x}^{+} \\
\hat{E}_{3, y}^{+}
\end{array}\right) e^{i k_{3, z}\left(z-d_{1}\right)} e^{i(\xi x+\eta y)} d \xi d \eta \\
& \mathbf{B}_{3, \tau}=\frac{1}{(2 \pi)^{2}} \int_{-\infty}^{+\infty} \int_{-\infty}^{+\infty}\left(\begin{array}{cc}
-\xi \eta / \omega k_{3, z} & -\left(k_{3, z}^{2}+\eta^{2}\right) / \omega k_{3, z} \\
\left(k_{3, z}^{2}+\xi^{2}\right) / \omega k_{3, z} & \xi \eta / \omega k_{3, z}
\end{array}\right) \\
& \quad \times\left(\begin{array}{c}
\hat{E}_{3, x}^{+} \\
\hat{E}_{3, y}^{+}
\end{array}\right) e^{i k_{3, z}\left(z-d_{1}\right)} e^{i(\xi x+\eta y)} d \xi d \eta .
\end{aligned}
$$

Полученные выражения для полей в рассматриваемых областях должны удовлетворять граничным условиям. Известно, что для гармонических полей на границах должны быть непрерывны тангенциальные компоненты напряженности электрического и индукции магнитного полей.

Рассмотрим границу первой и второй областей $z=0$. На ней в точках $(x, y, 0)$ непрерывность тангенциальных компонент электрического и магнитного полей можно записать в виде $E_{1, x}=E_{2, x}, E_{1, y}=E_{2, y}$, $B_{1, x} / \mu_{1}=B_{2, x} / \mu_{2}$ и $B_{1, y} / \mu_{1}=B_{2, y} / \mu_{2}$. Учитывая, что в точках границы $(x, y, 0)$ имеют место соотношения

$$
\begin{aligned}
& E_{1, x}=E_{1, x}^{+}+E_{1, x}^{-}, \quad E_{1, y}=E_{1, y}^{+}+E_{1, y}^{-}, \\
& E_{2, x}=E_{2, x}^{+}+E_{2, x}^{-}, \quad E_{2, y}=E_{2, y}^{+}+E_{2, y}^{-} \text {, } \\
& B_{1, x}=B_{1, x}^{+}+B_{1, x}^{-}, \quad B_{1, y}=B_{1, y}^{+}+B_{1, y}^{-}, \\
& B_{2, x}=B_{2, x}^{+}+B_{2, x}^{-}, \quad B_{2, y}=B_{2, y}^{+}+B_{2, y}^{-},
\end{aligned}
$$

после несложных преобразований, используя (13)-(16), из граничных условий получим следующие матричные уравнения:

$$
\left(\begin{array}{cc}
\mathbf{I} & \mathbf{I} \\
\mathbf{G}^{+} & \mathbf{G}^{-}
\end{array}\right)\left(\begin{array}{l}
\hat{E}_{1, x}^{+} \\
\hat{E}_{1, y}^{+} \\
\hat{E}_{1, x}^{-} \\
\hat{E}_{1, y}^{-}
\end{array}\right)=\left(\begin{array}{cc}
\mathbf{I} & e^{i k_{2, x} d_{1}} \mathbf{I} \\
\mathbf{G}_{2}^{+} & e^{i k_{2, x} d_{1}} \mathbf{G}_{2}^{-}
\end{array}\right)\left(\begin{array}{l}
\hat{E}_{2, x}^{+} \\
\hat{E}_{2, y}^{+} \\
\hat{E}_{2, x}^{-} \\
\hat{E}_{2, y}^{-}
\end{array}\right)
$$

или

$$
\left(\begin{array}{c}
\hat{E}_{1, x}^{+} \\
\hat{E}_{1, y}^{+} \\
\hat{E}_{1, x}^{-} \\
\hat{E}_{1, y}^{-}
\end{array}\right)=\left(\begin{array}{cc}
\mathbf{I} & \mathbf{I} \\
\mathbf{G}_{1}^{+} & \mathbf{G}_{1}^{-}
\end{array}\right)^{-1}\left(\begin{array}{cc}
\mathbf{I} & e^{i k_{2, z} d_{1}} \mathbf{I} \\
\mathbf{G}_{2}^{+} & e^{i k_{2, z} d_{1}} \mathbf{G}_{2}^{-}
\end{array}\right)\left(\begin{array}{l}
\hat{E}_{2, x}^{+} \\
\hat{E}_{2, y}^{+} \\
\hat{E}_{2, x}^{-} \\
\hat{E}_{2, y}^{-}
\end{array}\right),
$$

где I - единичная матрица $2 \times 2$, а остальные матрицы имеют следующий вид:

$$
\begin{aligned}
& \mathbf{G}_{1}^{+}=\left(\begin{array}{cc}
-\frac{\xi \eta}{\omega \mu_{1} k_{1, z}} & -\frac{k_{1, z}^{2}+\eta^{2}}{\omega \mu_{1} k_{1, z}} \\
\frac{k_{1, z}^{2}+\xi^{2}}{\omega \mu_{1} k_{1, z}} & \frac{\xi \eta}{\omega \mu_{1} k_{1, z}}
\end{array}\right), \quad \mathbf{G}_{1}^{-}=\left(\begin{array}{cc}
\frac{\xi \eta}{\omega \mu_{1} k_{1, z}} & \frac{k_{1, z}+\eta^{2}}{\omega \mu_{1} k_{1, z}} \\
-\frac{k_{1, z}^{2}+\xi^{2}}{\omega \mu_{1} k_{1, z}} & -\frac{\xi \eta}{\omega \mu_{1} k_{1, z}}
\end{array}\right), \\
& \mathbf{G}_{2}^{+}=\left(\begin{array}{cc}
-\frac{\xi \eta}{\omega \mu_{2} k_{2, z}} & -\frac{k_{2, z}^{2}+\eta^{2}}{\omega \mu_{2} k_{2, z}} \\
\frac{k_{1, z}^{2}+\xi^{2}}{\omega \mu_{2} k_{2, z}} & \frac{\xi \eta}{\omega \mu_{2} k_{2, z}}
\end{array}\right), \quad \mathbf{G}_{2}^{-}=\left(\begin{array}{cc}
\frac{\xi \eta}{\omega \mu_{2} k_{2, z}} & \frac{k_{2, z}+\eta^{2}}{\omega \mu_{2} k_{2, z}} \\
-\frac{k_{2, z}^{2}+\xi^{2}}{\omega \mu_{2} k_{2, z}} & -\frac{\xi \eta}{\omega \mu_{2} k_{2, z}}
\end{array}\right) .
\end{aligned}
$$

Аналогично рассмотрим границу второй и третьей областей $z=d_{1}$ (т.е. точки плоскости $\left.\left(x, y, d_{1}\right)\right)$ и запишем граничные условия в точках этой границы: $E_{2, x}=E_{3, x}$, $E_{2, y}=E_{3, y}, B_{2, x} / \mu_{2}=B_{3, x} / \mu_{3}$ и $B_{2, y} / \mu_{2}=B_{3, y} / \mu_{3}$. Используя выражения (15)-(18), получим следующие матричные уравнения:

$$
\left(\begin{array}{cc}
e^{i k_{2, z} d_{1}} \mathbf{I} & \mathbf{I} \\
e^{i k_{2, x} d_{1}} \mathbf{G}_{2}^{+} & \mathbf{G}_{2}^{-}
\end{array}\right)\left(\begin{array}{c}
\hat{E}_{2, x}^{+} \\
\hat{E}_{2, y}^{+} \\
\hat{E}_{2, x}^{-} \\
\hat{E}_{2, y}^{-}
\end{array}\right)=\left(\begin{array}{cc}
\mathbf{I} & 0 \\
\mathbf{G}_{3}^{+} & 0
\end{array}\right)\left(\begin{array}{c}
\hat{E}_{3, x}^{+} \\
\hat{E}_{3, y}^{+} \\
0 \\
0
\end{array}\right)
$$

или

$$
\left(\begin{array}{c}
\hat{E}_{2, x}^{+} \\
\hat{E}_{2, y}^{+} \\
\hat{E}_{2, x}^{-} \\
\hat{E}_{2, y}^{-}
\end{array}\right)=\left(\begin{array}{cc}
e^{i k_{2, z} d_{1}} \mathbf{I} & \mathbf{I} \\
e^{i k_{2, z} d_{1}} \mathbf{G}_{2}^{+} & \mathbf{G}_{2}^{-}
\end{array}\right)^{-1}\left(\begin{array}{cc}
\mathbf{I} & 0 \\
\mathbf{G}_{3}^{+} & 0
\end{array}\right)\left(\begin{array}{c}
\hat{E}_{3, x}^{+} \\
\hat{E}_{3, y}^{+} \\
0 \\
0
\end{array}\right)
$$

где

$$
\mathbf{G}_{3}^{+}=\left(\begin{array}{cc}
-\frac{\xi \eta}{\omega \mu_{3} k_{3, z}} & -\frac{k_{3, z}^{2}+\eta^{2}}{\omega \mu_{3} k_{3, z}} \\
\frac{k_{3, z}^{2}+\xi^{2}}{\omega \mu_{3} k_{3, z}} & \frac{\xi \eta}{\omega \mu_{3} k_{3, z}}
\end{array}\right) .
$$

Из матричных уравнений (19), (20) получаем

$$
\begin{aligned}
& \left(\begin{array}{c}
\hat{E}_{1, x}^{+} \\
\hat{E}_{1, y}^{+} \\
\hat{E}_{1, x}^{-} \\
\hat{E}_{1, y}^{-}
\end{array}\right)=\left(\begin{array}{cc}
\mathbf{I} & \mathbf{I} \\
\mathbf{G}_{1}^{+} & \mathbf{G}_{+}^{-}
\end{array}\right)^{-1}\left(\begin{array}{cc}
\mathbf{I} & e^{i k_{2, z} d_{1} \mathbf{I}} \\
\mathbf{G}_{2}^{+} & e^{i k_{2, z} d_{1}} \mathbf{G}_{2}^{-}
\end{array}\right) \\
& \times\left(\begin{array}{cc}
e^{i k_{2, z} d_{1} \mathbf{I}} & \mathbf{I} \\
e^{i k_{2, z} d_{1}} \mathbf{G}_{2}^{+} & \mathbf{G}_{2}^{-}
\end{array}\right)^{-1}\left(\begin{array}{cc}
\mathbf{I} & 0 \\
\mathbf{G}_{3}^{+} & 0
\end{array}\right)\left(\begin{array}{c}
\hat{E}_{3, x}^{+} \\
\hat{E}_{3, y}^{+} \\
0 \\
0
\end{array}\right),
\end{aligned}
$$


или

$$
\left(\begin{array}{c}
\hat{E}_{1, x}^{+} \\
\hat{E}_{1, y}^{+} \\
\hat{E}_{1, x}^{-} \\
\hat{E}_{1, y}^{-}
\end{array}\right)=\mathbf{M}\left(\begin{array}{c}
\hat{E}_{3, x}^{+} \\
\hat{E}_{3, y}^{+} \\
0 \\
0
\end{array}\right),
$$

где матрица $\mathbf{M}$ представляется в виде произведения трех матриц $\mathbf{M}=\mathbf{T}_{1} \mathbf{T}_{2} \mathbf{T}_{3}$, в котором

$$
\begin{gathered}
\mathbf{T}_{1}=\left(\begin{array}{cc}
\mathbf{I} & \mathbf{I} \\
\mathbf{G}_{1}^{+} & \mathbf{G}_{1}^{-}
\end{array}\right)^{-1}, \\
\mathbf{T}_{2}=\left(\begin{array}{cc}
\mathbf{I} & e^{i k_{2, z} d_{1}} \mathbf{I} \\
\mathbf{G}_{2}^{+} & e^{i k_{2, z} d_{1}} \mathbf{G}_{2}^{-}
\end{array}\right)\left(\begin{array}{cc}
e^{i k_{2, z} d_{1}} \mathbf{I} & \mathbf{I} \\
e^{i k_{2, z} d_{1}} \mathbf{G}_{2}^{+} & \mathbf{G}_{2}^{-}
\end{array}\right)^{-1}, \\
\mathbf{T}_{3}=\left(\begin{array}{cc}
\mathbf{I} & 0 \\
\mathbf{G}_{3}^{+} & 0
\end{array}\right) .
\end{gathered}
$$

Отметим, что обобщение формулы (21) на плоскослоистую структуру из $N$ слоев можно произвести по индукции, которая приводит к следующим выражениям:

$$
\left(\begin{array}{c}
\hat{E}_{1, x}^{+} \\
\hat{E}_{1, y}^{+} \\
\hat{E}_{1, x}^{-} \\
\hat{E}_{1, y}^{-}
\end{array}\right)=\mathbf{M}\left(\begin{array}{c}
\hat{E}_{N+2, x}^{+} \\
\hat{E}_{N+2, y}^{+} \\
0 \\
0
\end{array}\right),
$$

причем матрица $\mathbf{M}$ имеет вид $\mathbf{M}=\mathbf{T}_{1}\left(\prod_{m=2}^{N+1} \mathbf{T}_{m}\right) \mathbf{T}_{N+2}$, где

$$
\begin{gathered}
\mathbf{T}_{1}\left(\begin{array}{cc}
\mathbf{I} & \mathbf{I} \\
\mathbf{G}_{1}^{+} & \mathbf{G}_{1}^{-}
\end{array}\right), \\
\mathbf{T}_{m}=\left(\begin{array}{cc}
\mathbf{I} & e^{i k_{m, z} d_{m-1} \mathbf{I}} \\
\mathbf{G}_{m}^{+} & e^{i k_{m, z} d_{m-1}} \mathbf{G}_{m}^{-}
\end{array}\right)\left(\begin{array}{cc}
e^{i k_{m, z} d_{m-1} \mathbf{I}} & \mathbf{I} \\
e^{i k_{m, z} d_{m-1}} \mathbf{G}_{m}^{+} & \mathbf{G}_{m}^{-}
\end{array}\right)^{-1}, \\
\mathbf{T}_{N+2}=\left(\begin{array}{cc}
\mathbf{I} & 0 \\
\mathbf{G}_{N+2}^{+} & 0
\end{array}\right) .
\end{gathered}
$$

Отметим, что обычно интересуются задачей падения известной, заданной волны на плоскослоистую структуру. Тогда эту падающую волну можно разложить в ряд Фурье, и найти функции $\hat{E}_{1, x}^{+}(\xi, \eta), \hat{E}_{1, y}^{+}(\xi, \eta)$. Разделив матрицу М в (22) на четыре $2 \times 2$-матрицы $\mathbf{M}_{\mathrm{A}}, \mathbf{M}_{\mathrm{B}}, \mathbf{M}_{\mathrm{C}}$ и $\mathbf{M}_{\mathrm{D}}$ :

$$
\mathbf{M}=\left(\begin{array}{ll}
\mathbf{M}_{\mathrm{A}} & \mathbf{M}_{\mathrm{B}} \\
\mathbf{M}_{\mathrm{C}} & \mathbf{M}_{\mathrm{D}}
\end{array}\right),
$$

получим матричные уравнения

$$
\left(\begin{array}{c}
\hat{E}_{1, x}^{+} \\
\hat{E}_{1, y}^{+}
\end{array}\right)=\mathbf{M}_{\mathrm{A}}\left(\begin{array}{c}
\hat{E}_{N+2, x}^{+} \\
\hat{E}_{N+2, y}^{+}
\end{array}\right) \text {и }\left(\begin{array}{c}
\hat{E}_{1, x}^{-} \\
\hat{E}_{1, x}^{+}
\end{array}\right)=\mathbf{M}_{\mathrm{C}}\left(\begin{array}{c}
\hat{E}_{N+2, x}^{+} \\
\hat{E}_{N+2, y}^{+}
\end{array}\right),
$$

из которых по компонентам падающей волны $\hat{E}_{1, x}^{+}$и $\hat{E}_{1, y}^{+}$ можно найти компоненты прошедшей волны:

$$
\left(\begin{array}{c}
\hat{E}_{N+2, x}^{+} \\
\hat{E}_{N+2, y}^{+}
\end{array}\right)=\mathbf{M}_{A}^{-1}\left(\begin{array}{c}
\hat{E}_{1, x}^{+} \\
\hat{E}_{1, y}^{+}
\end{array}\right),
$$

и отраженной волны:

$$
\left(\begin{array}{c}
\hat{E}_{1, x}^{-} \\
\hat{E}_{1, y}^{-}
\end{array}\right)=\mathbf{M}_{\mathrm{C}} \mathbf{M}_{\mathrm{A}}^{-1}\left(\begin{array}{c}
\hat{E}_{1, x}^{+} \\
\hat{E}_{1, y}^{+}
\end{array}\right) .
$$

Тогда из (8) напряженности падающего и отраженного полных электрических полей будут определяться следующими формулами:

$$
\begin{aligned}
& \mathbf{E}_{1}^{i n}(x, y, z)= \frac{1}{(2 \pi)^{2}} \int_{-\infty}^{+\infty} \int_{-\infty}^{+\infty}\left(\begin{array}{cc}
1 & 0 \\
0 & 1 \\
-\xi / k_{1, z} & -\eta / k_{1, z}
\end{array}\right) \\
& \times\left(\begin{array}{c}
\hat{E}_{1, x}^{+} \\
\hat{E}_{1, y}^{+}
\end{array}\right) e^{i k_{1, z} z} e^{i(\xi x+\eta y)} d \xi d \eta, \\
& \mathbf{E}_{1}^{r e f}(x, y, z)= \frac{1}{(2 \pi)^{2}} \int_{-\infty}^{+\infty} \int_{-\infty}^{+\infty}\left(\begin{array}{cc}
1 & 0 \\
0 & 1 \\
\xi / k_{1, z} & \eta / k_{1, z}
\end{array}\right) \\
& \quad \times \mathbf{M}_{\mathrm{C}} \mathbf{M}_{\mathrm{A}}^{-1}\left(\begin{array}{c}
\hat{E}_{1, x}^{+} \\
\hat{E}_{1, y}^{+}
\end{array}\right) e^{-i k_{1, z} z} e^{i(\xi x+\eta y)} d \xi d \eta,
\end{aligned}
$$

а напряженность прошедшего электрического поля по аналогии с (10) будет определяться формулой

$$
\begin{aligned}
& \mathbf{E}_{N+2}^{\text {trans }}(x, y, z)=\frac{1}{(2 \pi)^{2}} \int_{-\infty}^{+\infty} \int_{-\infty}^{+\infty}\left(\begin{array}{cc}
1 & 0 \\
0 & 1 \\
\xi / k_{N+2, z} & -\eta / k_{N+2, z}
\end{array}\right) \\
& \times \mathbf{M}_{\mathrm{A}}^{-1}\left(\begin{array}{c}
\hat{E}_{1, x}^{+} \\
\hat{E}_{1, y}^{+}
\end{array}\right) e^{i k_{N+2, z}\left(z-d_{t o t}\right)} e^{i(\xi x+\eta y)} d \xi d \eta,
\end{aligned}
$$

где $d_{t o t}=\sum_{i=1}^{N} d_{i}-$ полная толщина плоскослоистой структуры.

C помощью соотношений (11) и (12) легко можно выписать и выражения для индукции падающего, отраженного и прошедшего магнитных полей (для экономии места мы не будем здесь выписывать эти выражения).

\section{Задача падения конической электромагнитной волны ограниченной апертуры на плоскослоистую структуру}

Рассмотрим (рис. 3) падение на плоскослоистую структуру конической волны $\mathbf{E}$, которую в плоскости 


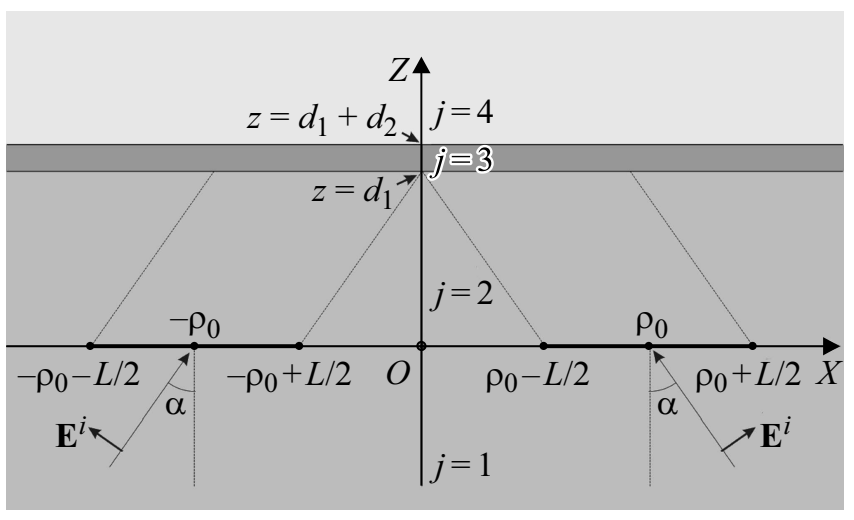

Рис. 3. Геометрия падения конической волны. Область $j=1$ - полупространство призмы; $j=2$ - вспомогательная пленка толщины $d_{1}$ из того же материала, что и призма; $j=3$ - металлическая пленка толщины $d_{2} ; j=4-$ свободное пространство за металлической пленкой.

$z=0$ определим функцией

$$
\begin{aligned}
& \mathbf{E}_{1}^{+}(x, y, 0)=e^{i\left(-k_{1}\left(\sqrt{x^{2}+y^{2}}-\rho_{0}\right) \sin \alpha+k_{1} z \cos \alpha\right)} \\
& \quad \times\left(-\frac{x \cos \alpha}{\sqrt{x^{2}+y^{2}}} \mathbf{e}_{x}-\frac{y \cos \alpha}{\sqrt{x^{2}+y^{2}}} \mathbf{e}_{y}-\sin \alpha \mathbf{e}_{z}\right),
\end{aligned}
$$

где $k_{1}=n_{1} \omega / c-$ волновое число в первой среде, $c$ - скорость света в вакууме, $n_{1}=\sqrt{\varepsilon_{1}}-$ показатель преломления в первой среде, $\mathbf{e}_{x}, \mathbf{e}_{y}, \mathbf{e}_{z}-$ как и ранее, единичные векторы в направлениях осей $X, Y$ и $Z$ соответственно, $\alpha$ - угол падения волны на плоскость $z=0$ в локально-плоском приближении. Вектор напряженности электрического поля волны предполагается ориентированным всегда в плоскости падения ( $p$-поляризация). На рис. 3 показана геометрия задачи в разрезе плоскостью $X Z$. Рассматриваемые области задачи мы пронумеруем следующим образом: $j=1$ - полупространство призмы; $j=2$ - вспомогательная пленка из того же материала, что и призма, толщины $d_{1} ; j=3-$ металлическая пленка толщины $d_{2} ; j=4-$ свободное пространство за металлической пленкой. В такой постановке задачи мы задаем поле падающей конической волны внутри призмы на некотором расстоянии $d_{1}$ от металлической пленки.

Отметим, что экспериментальные методы создания таких конических волн с радиальной поляризацией хорошо развиты [14-16].

Пусть при $z=0$ апертура волны ограничена, причем амплитуда волны (26) постоянна (в локально-плоском приближении амплитуда равна единице) и отлична от нуля только в кольце $\rho \in\left[\rho_{0}-L / 2, \rho_{0}+L / 2\right]$ (где $\rho=\sqrt{x^{2}+y^{2}}$ и $\rho_{0}>L 2$, причем центральная окружность этого кольца имеет радиус $\rho_{0}$ ). Тогда компоненты
Фурье этой волны при $z=0$ можно зависать в виде

$$
\begin{aligned}
& \hat{E}_{1, x}^{i n}(\xi, \eta)=-\iint \frac{x \cos \alpha}{\sqrt{x^{2}+y^{2}}} \\
& \times e^{-k_{1}\left(\sqrt{x^{2}+y^{2}}-\rho_{0}\right) \sin \alpha} e^{-i(\xi x+\eta y)} d x d y, \\
& \hat{E}_{1, y}^{i n}(\xi, \eta)=-\iint \frac{y \cos \alpha}{\sqrt{x^{2}+y^{2}}} \\
& \quad \times e^{-k_{1}\left(\sqrt{x^{2}+y^{2}}-\rho_{0}\right) \sin \alpha} e^{-i(\xi x+\eta y)} d x d y .
\end{aligned}
$$

Интегрирование в (27) и (28) производится по указанному кольцу. Перейдем в (27) к полярным координатам $(\rho, \varphi)$, тогда

$$
\begin{aligned}
& \hat{E}_{1, x}^{i n}(\xi, \eta)=-\int_{0}^{2 \pi} \int_{\rho_{0}-L / 2}^{\rho_{0}+L / 2} \frac{\rho \cos \varphi \cos \alpha}{\rho} e^{-i k_{1}\left(\rho-\rho_{0}\right) \sin \alpha} \\
& \times e^{-i(\xi \rho \cos \varphi+\eta \rho \sin \varphi)} \rho d \rho d \varphi=-\cos \alpha e^{i k_{1} \rho_{0} \sin \alpha} \\
& \times \int_{0}^{2 \pi} \mathscr{F}\left(-k_{1} \sin \alpha-\xi \cos \varphi-\eta \sin \varphi\right) \cos \varphi d \varphi,
\end{aligned}
$$

где

$$
\begin{aligned}
\mathscr{F}(\chi)= & \int_{\rho_{0}-L / 2}^{\rho_{0}+L / 2} e^{i \rho \chi} \rho d \rho=\frac{e^{i \chi \rho_{0}}}{\chi}\left[2 \rho_{0} \sin (\chi L / 2)\right. \\
& +i((2 / \chi) \sin (\chi L / 2))-L \cos (\chi L / 2)] .
\end{aligned}
$$

Заметим, что

$$
\xi \cos \varphi+\eta \sin \varphi=\sqrt{\xi^{2}+\eta^{2}} \cos (\varphi-\vartheta),
$$

где $\vartheta=\operatorname{arctg}(\eta / \xi)$. Отсюда получаем

$$
\begin{aligned}
& -k_{1} \sin \alpha-\xi \cos \varphi-\eta \sin \varphi \\
& =-k_{1} \sin \alpha-\sqrt{\xi^{2}+\eta^{2}} \cos (\varphi-\vartheta)
\end{aligned}
$$

и выражение (29) примет вид

$$
\begin{aligned}
& \hat{E}_{1, x}^{i n}(\xi, \eta)=-\cos \alpha e^{i k_{1} \rho_{0} \sin \alpha} \\
& \times \int_{0}^{2 \pi} \mathscr{F}\left(-k_{1} \sin \alpha-\sqrt{\xi^{2}+\eta^{2}} \cos (\varphi-\vartheta)\right) \cos \varphi d \varphi .
\end{aligned}
$$

Аналогично из (28) получаем

$$
\begin{aligned}
\hat{E}_{1, y}^{i n}= & -\cos \alpha e^{i k_{1} \rho_{0} \sin \alpha} \int_{0}^{2 \pi} \mathscr{F}\left(-k_{1} \sin \alpha\right. \\
& \left.-\sqrt{\xi^{2}+\eta^{2}} \cos (\varphi-\vartheta)\right) \sin \varphi d \varphi .
\end{aligned}
$$

Подставляя (30) и (31) в уравнения (24) и (25), можно найти электрическое поле отраженной волны (24) в первой среде и электрическое поле прошедшей волны (25) в полупространстве за плоскослоистой структурой. 


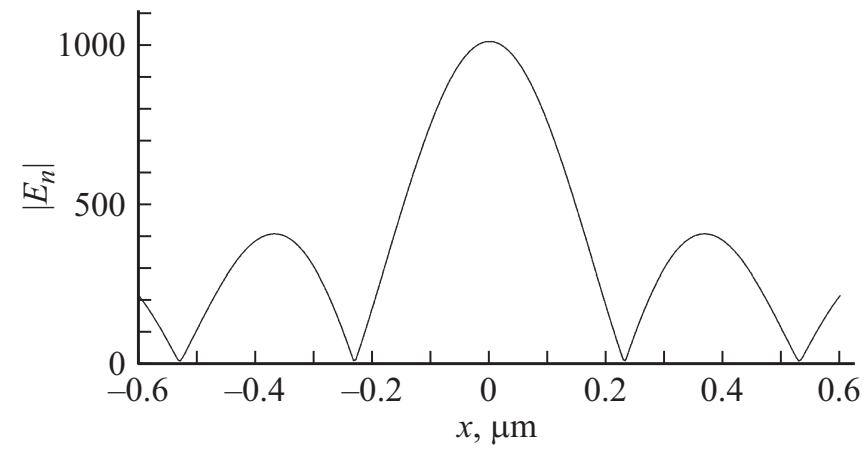

Рис. 4. Распределение амплитуды нормальной компоненты напряженности электрического поля $\left|E_{n}\right|$ в центре интерференционной картины радиально сходящейся поверхностной плазмонной волны на свободной границе золотой пленки. Величины $\left|E_{n}\right|$ нормированы на амплитуду падающего поля в призме при $z=0$.

\section{Вычисление сфокусированного поля на свободной границе золотой пленки}

Рассмотрим возбуждение конической волной (26) поверхностных плазмонных волн на свободной поверхности металлической пленки плоскослоистой структуры, показанной на рис. 3. Предполагается, что пленка 3 золотая, а длина падающей волны в вакууме $\lambda=633 \mathrm{~nm}$. Коническая волна сходится к плоскости $z=d_{1}=\left(\rho_{0}-L / 2\right) \operatorname{ctg} \alpha$, на которой помещена металлическая пленка. Указанное значение $d_{1}=\left(\rho_{0}-L / 2\right) \operatorname{ctg} \alpha$ выбрано таким образом, чтобы на этом расстоянии крайние внутренние лучи конической волны сходились бы к оси системы в точку $x=0$. Для определенности примем, что $L=200 \mu \mathrm{m}, \rho_{0}=2 L$, показатель преломления призмы $n_{1}=1.6$ (т.е. $\varepsilon_{1}=1.6^{2}$ ). Параметры сред, показанных на рис. 3, на рабочей длине волны представлены в таблице.

Значение угла падения $\alpha$ принималось равным $41.0^{\circ}$. Это значение $\alpha$ и геометрия падающей волны соответствуют наилучшему возбуждению поверхностной плазмонной волны на поверхности золотой пленки, если падающая волна плоская с неограниченной апертурой.

Было вычислено распределение электрического поля на свободной поверхности металлической пленки $\left(z=d_{t o t}=d_{1}+d_{2}\right)$ по формуле $(25)$. В настоящей работе нас будет интересовать электронная эмиссия со свободной поверхности металлической пленки, которая определяется нормальной составляющей электрического поля (z-составляющей). На рис. 4 представлены результаты расчетов распределения модуля $z$-составляющей напряженности электрического поля (нормальной составляющей) в области фокуса, т.е. точки $(x, y, z)=\left(0,0, d_{1}+d_{2}\right)$, на поверхности золотой пленки (таблица).

Как видно из рис. 4, в области фокуса на поверхности пленки наблюдается максимум нормальной составля- ющей напряженности электрического поля $\left|E_{n}\right|$. Электрическое поле симметрично относительно поворотов вокруг оси $Z$, поэтому на рисунке показана зависимость только вдоль оси $X$. В этом смысле можно записать нормальную компоненту комплексного поля в виде функции от расстояния $r$ от центра фокального распределения вдоль поверхности пленки как $E_{n}(r)=\left.E_{z}\left(x, d_{1}+d_{2}\right)\right|_{x=r}$.

Особо следует отметить большое усиление амплитуды поля в максимуме фокуса - 1010 в единицах амплитуды падающей в призме волны в плоскости $z=0$. Такое большое усиление поля определяется следующими причинами:

- ширина падающей волны $L=200 \mu \mathrm{m}$ в перпендикулярном оси направлении выбиралась достаточно большой, чтобы амплитуда поверхностной волны достигала значения, близкого к максимальному значению [3];

- угол падения $41.0^{\circ}$ соответствует оптимальному углу возбуждения поверхностной волны в плоской задаче с ограниченным падающим пучком [3];

- точка схождения внутренних лучей падающей волны к внутренней поверхности пленки $(x, y, z)=$ $=\left(0,0, d_{1}\right)$ соответствует максимальному полю поверхностной волны на другой стороне пленки, исходя из результатов решения плоской задачи [3];

- электрическое поле падающей волны поляризовано в плоскости падения (в плоскости, проходящей через ось задачи $Z$ ), что обеспечивает возбуждение только радиально сходящейся поверхностной волны с той же симметрией.

Расчеты показали, что если менять расстояние $d_{1}$, то максимальное значение поля в фокусе на свободной поверхности пленки слабо меняется при изменении $d_{1}$ в окрестности значения $\left(\rho_{0}-L / 2\right) \operatorname{ctg} \alpha$. Практически здесь фокальное поле равно максимальному значению. Поэтому настройка на фокальный максимум полученной системы не должна представлять проблемы.

\section{Вычисление тока электронной эмиссии из окрестности фокуса со свободной границы золотой пленки}

Термополевая электронная эмиссия с поверхности металла исследовалась с помощью подхода, изложенного в работах $[17,18]$. Плотность тока $j$ с поверхности металла вычислялась по известной формуле:

$$
j=\frac{4 \pi e m k T}{h^{3}} \int_{0}^{+\infty} D\left(\mathscr{E}_{n}\right) \ln \left(1+\exp \left[-\left(\mathscr{E}_{n}-\mathscr{E}_{F}\right) / k T\right]\right) d \mathscr{E}_{n}
$$

где $e, m$ - заряд и масса электрона, $k$ - постоянная Больцмана, $h$ - постоянная Планка, $T-$ абсолютная температура катода, $\mathscr{E}_{F}-$ энергия Ферми металла, $\mathscr{E}_{n}-$ кинетическая энергия электрона в направлении, 
Параметры сред

\begin{tabular}{c|c|c|c}
\hline $\begin{array}{c}\text { Порядковый } \\
\text { номер } \\
\text { среды, } j\end{array}$ & Название слоя & $\begin{array}{c}\text { Относительная } \\
\text { диэлектрическая } \\
\text { проницаемость }\end{array}$ & Толщина пленки \\
\hline 1 & Призма & $1.6^{2}$ & $\begin{array}{c}\text { Полубесконечная среда } \\
2\end{array}$ \\
3 & Слой материала призмы & $1.6^{2}$ & $d_{1}=\left(\rho_{0}-L / 2\right) \operatorname{ctg} \alpha=357 \mu \mathrm{m}$ \\
$48.7 \mathrm{~nm}$
\end{tabular}

перпендикулярном поверхности, $D\left(\mathscr{E}_{n}\right)$ - квантовомеханический коэффициент прохождения электрона с энергией потенциального барьера на границе металла и окружающего вакуума.

Потенциальный барьер $V(\xi)$ на границе металла для выходящих из него электронов описывался барьером Шоттки с потенциальной энергией электрона

$$
V(\xi)=\left\{\begin{array}{ll}
W+\mathscr{E}_{F}-e^{2} / 16 \pi \varepsilon_{0} \xi-e E_{n} \xi, & \xi>0 \\
0, & \xi \leq \xi_{l}
\end{array},\right.
$$

где $\xi-$ координата по оси, перпендикулярной поверхности металла; $\varepsilon_{0}$ - диэлектрическая проницаемость вакуума; $W$ - работа выхода из металла; $E_{n}$ - напряженность нормальной компоненты внешнего электрического поля на поверхности металла; $\xi_{l}-$ меньший из корней уравнения $W+\mathscr{E}_{F}-e^{2} / 16 \pi \varepsilon_{0} \xi-e E_{s} \xi=0$.

Квантово-механическая задача прохождения через барьер Шоттки (33) и нахождения $D\left(\mathscr{E}_{n}\right)$ сводится к решению уравнения Шредингера для падающей на барьер волны единичной амплитуды. Она решалась численным методом, подробно рассмотренным в работе [17]. Поэтому для экономии места в настоящей работе не обсуждается.

Была решена задача нахождения зависимости плотности тока $j$ термополевой эмиссии для золота $\left(W=5.1 \mathrm{eV}, \mathscr{E}_{F}=5.5 \mathrm{eV}\right) \quad[19]$ при температуре $T=300 \mathrm{~K}$ от нормальной компоненты напряженности электрического поля на поверхности металла $E_{n}$. Расчеты проводились по формуле (32), график зависимости $j=j\left(E_{n}\right)$ показан на рис. 5.

Верхняя граница напряженности поля при вычислениях ограничивалась значением примерно $E_{\max }=1.2 \cdot 10^{9} \mathrm{~V} / \mathrm{m}$. При этом значении напряженности поля происходит переход от охлаждения поверхности (из-за эмиссии электронов) к эмиссионному нагреву поверхности, причем нагрев возникает лавинообразно из-за положительной обратной связи по температуре, что приводит к разрушению или изменению формы эмиссионной поверхности. Указанное значение напряженности поля $1.2 \cdot 10^{9} \mathrm{~V} / \mathrm{m}$, соответствующее переходу от охлаждения к нагреву, было найдено методом работы [18] для золотой поверхности $\left(W=5.1 \mathrm{eV}, \mathscr{O}_{F}=5.5 \mathrm{eV}\right)$ при температуре $T=300 \mathrm{~K}$.

Функцию $E_{n}(r)$, полученную в предыдущем разделе и нормированную на амплитуду поля падающей волны в

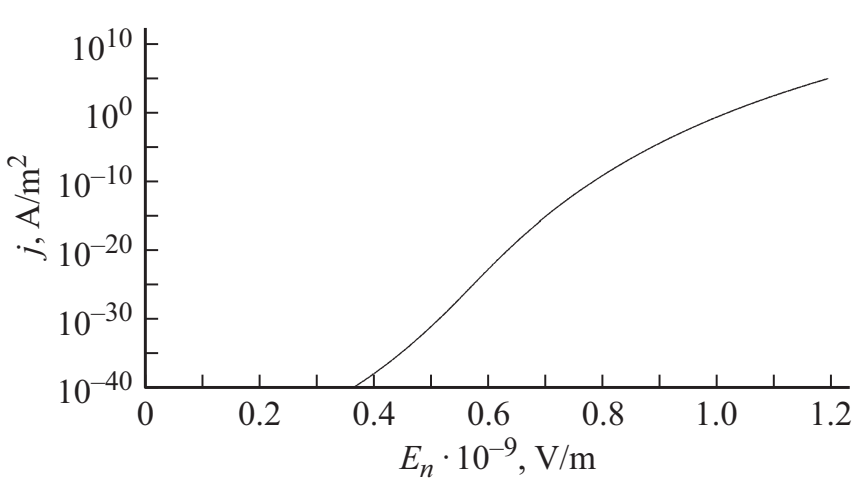

Рис. 5. Плотность тока термополевой электронной эмиссии при температуре $T=300 \mathrm{~K}$ с плоской границы золота $\left(W=5.1 \mathrm{eV}, \mathscr{E}_{F}=5.5 \mathrm{eV}\right)$ в зависимости от напряженности электрического поля на поверхности $E_{n}$.

призме, для нахождения поля эмиссии мы нормируем так, чтобы амплитуда поля в фокальном максимуме была равна некоторому значению $E_{m}$. То есть будем рассматривать распределение поля $E_{n}(r)$, определяемое как

$$
E_{n}(r)=E_{m}\left(E_{n}(r) /\left|E_{n}(0)\right|\right) .
$$

Исходя из полученной зависимости $j$ от нормальной компоненты напряженности электрического поля, зная распределение поля $E_{n}(r)$, можно найти полный ток эмиссии, усредненный по периоду световой волны, из фокального пятна на свободной поверхности золотой пленки по формуле

$$
J\left(E_{m}\right)=\frac{1}{T} \int_{0}^{T}\left(\int_{0}^{+\infty} j\left(\operatorname{Re}\left[E_{n}(r) e^{-i \omega t}\right]\right) 2 \pi r d t\right) d t,
$$

где $\omega=2 \pi c / \lambda-$ циклическая частота светового поля, $c$ - скорость света в вакууме, $\lambda$ - длина волны рассматриваемой электромагнитной световой волны в вакууме, $T=\lambda / c$ - период световой волны, $\operatorname{Re}-$ действительная часть выражения в квадратных скобках. В формуле (34) плотность тока $j$ при запирающем электронную эмиссию направлении электрического поля полагалась равной нулю.

Зависимость полного тока $J$ из фокального пятна от амплитуды волны в максимуме фокального пятна $E_{m}$ показана на рис. 6, $a$. Эта зависимость 

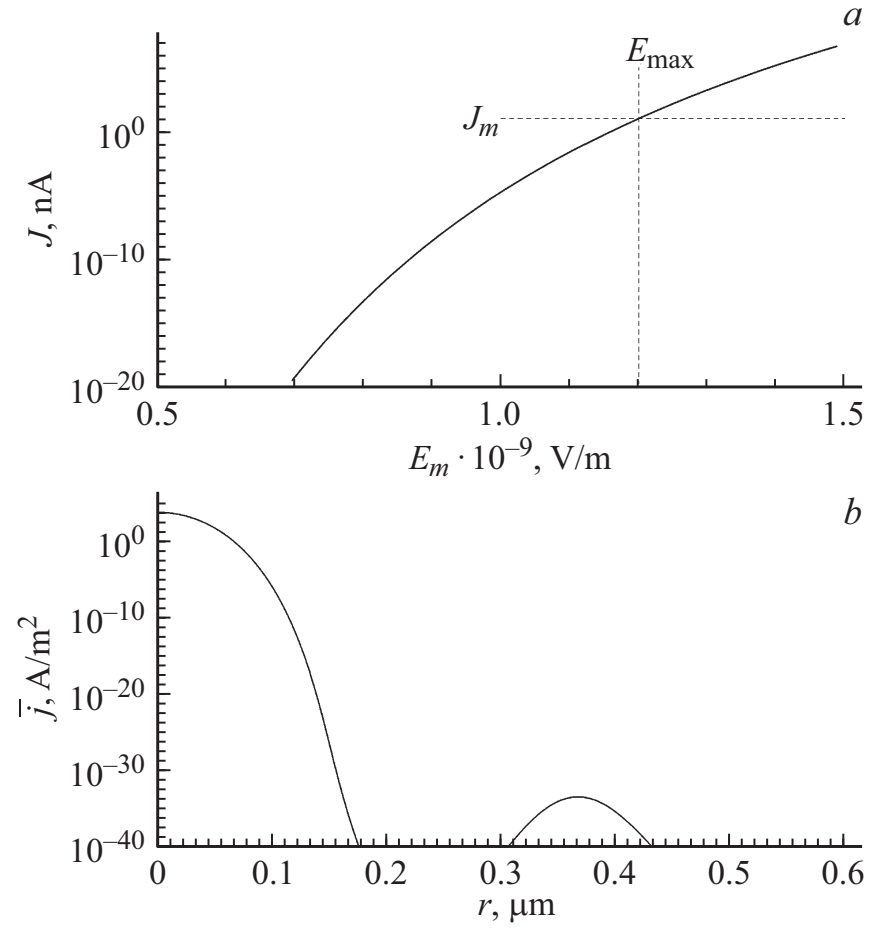

Рис. 6. (a) Зависимость полного тока $J$ из фокального пятна от амплитуды волны в максимуме фокального пятна $E_{m}$; (b) Зависимость средней плотности тока $\bar{j}$ от расстояния от центра фокального распределения $r$.

очень резкая, указана граница неразрушающего тока $J_{m}=9.84 \cdot 10^{-12} \mathrm{~A}=9.84 \mathrm{pA}$ при амплитуде электрического поля в максимуме $E_{m}=E_{\max }=1.2 \cdot 10^{9} \mathrm{~V} / \mathrm{m}$.

Зависимость средней плотности тока

$$
\bar{j}(r)=\frac{1}{T} \int_{0}^{T} j\left(\operatorname{Re}\left[E_{n}(r) e^{-i \omega t}\right]\right) d t
$$

от расстояния от центра фокального распределения $r$ показана в логарифмическом масштабе на рис. $6, b$. Видно, что, из-за очень резкой зависимости $j=j\left(E_{n}\right)$ электроны эмитируются практически только из центральной области фокуса. Было найдено, что 99.9\% электронов исходят из круга радиуса $59.3 \mathrm{~nm}$, а $66.7 \%$ из круга радиуса $24.3 \mathrm{~nm}$ и $50 \%$ из круга радиуса $19.4 \mathrm{~nm}$. Важно, что интерференционные круги вокруг центрального фокального пятна практически не дают вклад в эмиссионный ток.

\section{Заключение}

Предложен теоретический метод исследования электронной термополевой эмиссии из фокальной области сфокусированной радиально сходящейся поверхностной плазмонной волны на свободной поверхности золотой пленки. Найдены фокальные распределения электрического поля и значения усиления электрического поля в фокусе. На основе этого распределения проведена оценка среднего по времени тока электронной эмиссии в зависимости от амплитуды нормальной составляющей напряженности электрического поля волны в центре фокального распределения. Показано, что основная часть эмиссионного тока исходит из области вокруг центра фокуса диаметром порядка десятой части рабочей длины волны в вакууме, причем из окружающих центральный максимум дифракционных колец исходит пренебрежимо малая часть эмиссионного тока.

\section{Список литературы}

[1] Петрин А.Б. // Квант. электрон. 2019. Т. 49. № 3. С. 258.

[2] Петрин А.Б. // Опт. и спектр. 2019. Т. 127. В. 4. С. 654.

[3] Петрин А.Б. // Опт. и спектр. 2018. Т. 125. В. 6. С. 830.

[4] Петрин А.Б. // Опт. и спектр. 2019. Т. 126. В. 3. С. 350.

[5] Racz P., Irvine S.E., Lenner M. et al. // Appl. Phys. Lett. 2011. V. 98. N 11. P. 111116.

[6] Welsh G.H., Wynne K. // Opt. Express. 2009. V. 17. N 4. P. 2470

[7] Piliarik M., Homola J. // Opt. Express. 2009. V. 17. N 19. P. 16505.

[8] Liedberg B., Nylander C., Lundstrom I. // Sensors and Actuators. 1983. V. 4. P. 299.

[9] Liedberg B., Nylander C., Lundstrom I. // Biosens. Bioelectron. 1995. V. 10. P. i-ix.

[10] Новотный Л., Хехт Б. Основы нанооптики. М.: Физматлит, 2009. $484 \mathrm{c}$.

[11] Garabedian R., Gonzalez C., Richards J. et al. // Sensors and Actuators A. 1994. V. 43. P. 202.

[12] Sommerfeld A. // Annalen der Physik. 1909. V. 333. N 28. P. 665.

[13] Бреховских Л.М. Волны в слоистых средах. М.: Наука, 1973.

[14] Налимов А.Г., О’Фаолейн Л., Стафееев С.С., Шанина М.И., Котляр В.В. // Компьютерная оптика. 2014. Т. 38. № 2. C. 229.

[15] Стабеев С.С., Котляр В.В. // Компьютерная оптика. 2017. T. 41. № 2. C. 147.

[16] Ma Y. // Opt. Rev. 2012. V. 19. N 2. P. 39.

[17] Петрин А.Б. // ЖЭТФ. 2009. Т. 136. № 2(8). С. 369.

[18] Петрин А.Б. // Теплофизика высоких температур. 2011. T. 49. № 4. C. 492.

[19] Физические величины: Справочник. М.: Энергоатомиздат, 1991. 